Späte atone Uterusnachblutung

\title{
Stärkste vaginale Blutung drei Wochen postpartal - junge Frau droht zu verbluten!
}

M. Sroka, Institut für Anästhesiologie und operative Intensivmedizin, Klinikum Stadt Hanau (Direktor: Prof. Dr. P. M. Osswald) M. Franke, Frauenklinik, Klinikum Stadt Hanau (Direktor: Prof. Dr. H.-H. Zippel)

NOTFALLMEDIZIN 2003; 29: 419-421

Die vorliegende Kasuistik stellt den Fall einer späten atonen Uterusnachblutung mit einem sich daraus entwickelnden hämorrhagischen Schock dar. Die Patientin alarmierte erst nach einigen Tagen und nach immer stärker werdender vaginaler Blutung mit Kollaps den Rettungsdienst. Offensichtlich folgte der ambulanten Geburt keine intensive postpartale Nachbetreuung durch eine Hebamme. Dieser wäre ein sich schlecht zurückbildender Uterus aufgefallen. Auch Veränderungen beim Wochenfluss wären richtungsweisend gewesen und hätten eine frühzeitige gynäkologische Untersuchung veranlasst.

n den letzten Monaten wurde

in notfallmedizinischen Fachzeit-

schriften viel über das „Akute Abdomen“ aus gynäkologischer Sicht und über „oft mitgeführte und doch nie gebrauchte Medikamente“ in der Geburtshilfe berichtet. Parallel zur Veröffentlichung dieser Beiträge ereignete sich folgender Fall, den wir im Rahmen einer Kasuistik aufarbeiten wollen.

In der Nacht des 07. März 2003 wird der diensthabende gynäkologische Arzt von den Schwestern der zentralen Notaufnahme informiert, dass in Kürze der Notarztwagen (NAW) mit einer Patientin mit starker vaginaler Blutung eintreffe. Der Dienstarzt begibt sich daraufhin in die zentrale Notaufnahme, um dort das Eintreffen des NAW zu erwarten. Dieser trifft gegen 02:30 Uhr ein.

\section{Anamnese}

Der Notarzt übergibt eine 26jährige Patientin mit stärkster anhaltender vaginaler Blutung im hämorrhagischen Schock. Anamnestisch berichtet er: Zustand nach Spontanpartus (ambulant) vor drei Wochen mit Damm- und Labienriss. Seit gestern immer weiter zunehmende vaginale Blutung mit Kollaps heute Nacht.

\section{Befund}

Im Rahmen der rettungsdienstlichen Erstversorgung wurden bei einem systolischen Blutdruck von 60 $\mathrm{mmHg}$ ein großlumiger periphervenöser Zugang (ID 2,2 mm) angelegt und bis zum Eintreffen in die Klinik 1000 ml Kochsalzlösung sowie $500 \mathrm{ml}$ HAES rasch infundiert. Vor Einleitung dieser Infusionstherapie wurde vom Notarzt ein komplettes Labor, inklusive Kreuzblut abgenommen. Bei Übergabe ist die Patientin ansprechbar, zu allen Qualitäten orientiert, jedoch zentralisiert. Der Blutdruck beträgt 90/60 $\mathrm{mmHg}$, der Puls wird mit 130/min gemessen. Die periphere Sauerstoffsättigung beträgt $98 \%$. Die grob orientierende Erstuntersuchung durch den gynäkologischen Dienstarzt ergibt folgende Befunde:

- Abdomen weich

- Uterus schlecht kontrahiert (knapp unter Nabelniveau)

- frischer vaginaler Blut- und Koagelabgang

- keine Temperatur.

Sonographisch zeigt sich orientierend eine Uteruslänge von $13 \mathrm{~cm}$ und ein hoch aufgebautes Endometrium (Uterusschleimhaut) mit inhomogenen Reflexen.

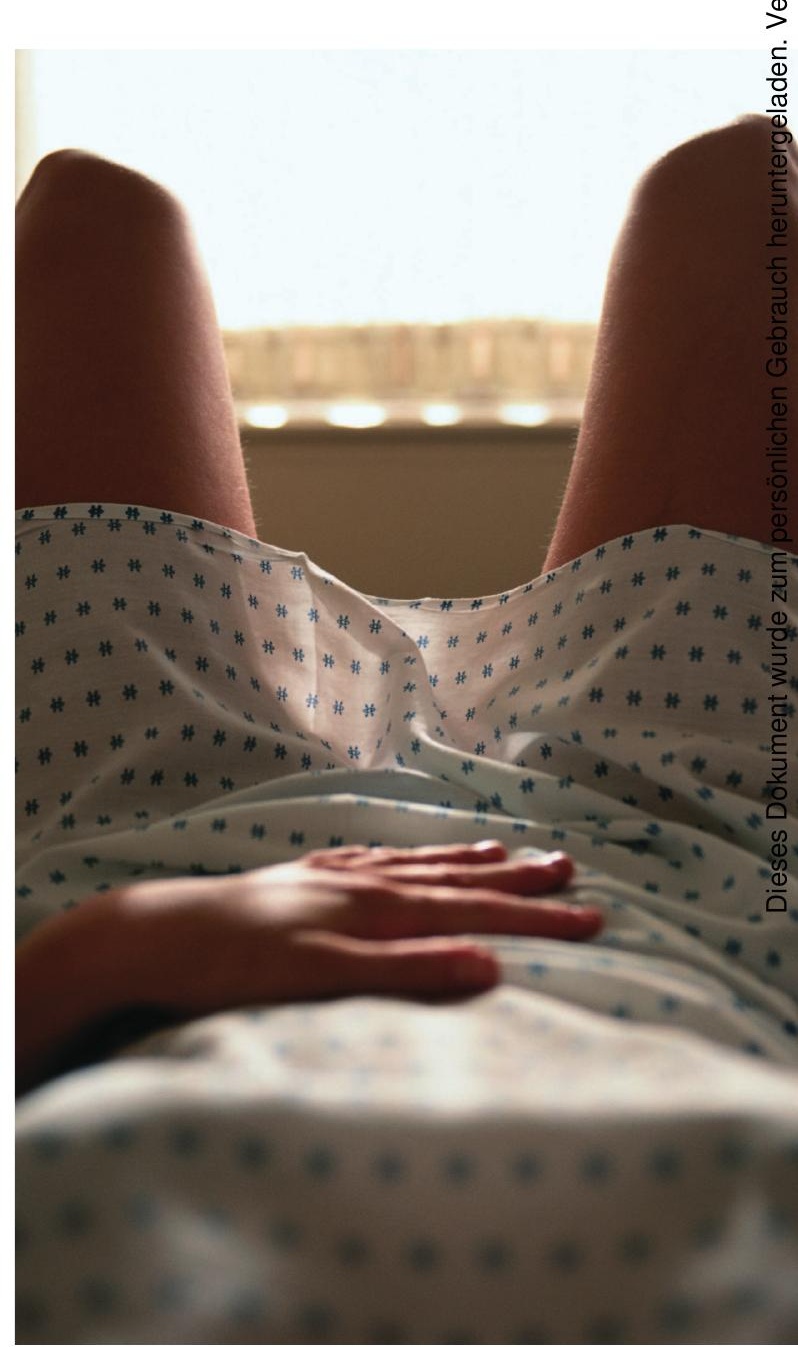




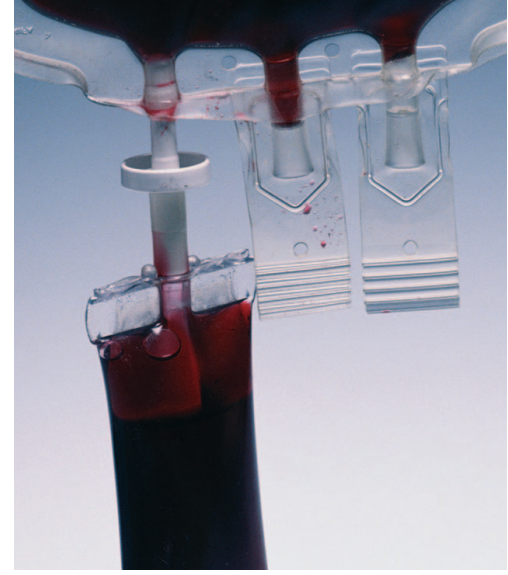
Es erfolgen noch die erneute Laborblutentnahme, sowie die Kreuzung von sechs Erythrozytenkonzentraten (EK's).

\section{Therapie}

Um 03:15 Uhr erfolgt im OP die Übergabe der Patienten an die Anästhesie. Dort wird unmittelbar die Narkose im Sinne einer Rapid Sequence Induction eingeleitet. Diese gestaltet sich problemlos. Im weiteren Verlauf werden noch mehrere periphervenöse Zugänge (ID 2,2 und $1,5 \mathrm{~mm}$ ) angelegt. Bei fortbestehender Blutung erhält die Patientin bis 04:00 Uhr weitere $2500 \mathrm{ml}$ Ringerlösung, sowie $500 \mathrm{ml}$ HAES 6\%. Unter dieser Therapie ist die Patientin zu jedem Zeitpunkt kreislaufstabil. Zur Tonisierung des Uterus werden initial 3 I.E. Orastin ${ }^{\circledR}$ sowie im weiteren Verlauf 0,2 mg Methergin ${ }^{\circledR}$ verabreicht. Parallel erfolgt die operative Sanierung im Sinne einer Nachcurretage. Hierbei werden größere Mengen an Plazentaresten entfernt. Nachdem sich unter der bisherigen Therapie der Uterus noch immer nicht ausreichend kontrahiert hat, wird zur weiteren Tonisierung ein Nalador ${ }^{\circledR}$-Perfusor angeordnet, sowie eine Tamponade eingebracht. Unter diesem Regime kommt es zum Sistieren der Blutung. Neben dieser kausalen Therapie erhält die Patientin intraoperativ eine AntibiotikaProphylaxe mit 1,5 g Cefuroxim i.v. Kurz nach OP-Ende gegen 04:00 Uhr kann die Patientin extubiert werden. Die Patientin wird daraufhin in den
Aufwachraum verlegt und dort weiter engmaschig überwacht, da eine Übernahme auf die Intensivstation zu diesem Zeitpunkt nicht möglich ist. In Absprache mit dem gynäkologischen Oberarzt wird als untere ein $\mathrm{Hb}$-Wert von $5 \mathrm{~g} / \mathrm{dl}$ vereinbart. Die inzwischen eingetroffenen Laborwerte zeigt Tabelle 1 .

Bei weiter sinkenden Werten und objektiver Beeinträchtigung der Patientin wird nach erneuter Rücksprache mit der Patientin die Indikation zur Fremdblutgabe gestellt. Die Patientin erhält insgesamt 3 EK's und 1 Fresh Frozen Plasma (FFP), zusätzlich an die Urinausscheidung adaptiert Ringerlösung. Die postoperative Schmerztherapie wird mit zwei Bolusgaben von Dipidolor ${ }^{\circledR}$ (jeweils 3,75 mg) und Metamizol sichergestellt. In den folgenden Stunden stabilisiert sich der Zustand der Patientin weiter. Auch die klinische Beeinträchtigung bildet sich rasch zurück. Nach insgesamt sechs Stunden kann die Patientin mit stabilen Kreislaufwerten auf die Normalstation verlegt werden.

Am ersten postoperativen Tag wird unter Op-Bereitschaft die eingebrachte Tamponade entfernt. Die nächsten Tage gestalten sich vollkommen problemlos. Am vierten postoperativen Tag drängt die Patientin auf schnellstmögliche Entlassung. Letztlich verlässt sie trotz intensiver Gespräche am Abend des 11.02.2003 gegen ärztlichen Rat die Klinik. Grenze für die Gabe von Fremdblut

\section{- Diskussion}

Die vorliegende Kasuistik stellt den Fall einer späten atonen Uterusnachblutung mit sich daraus entwickelndem hämorrhagischen Schock dar. Diese schwere Verlaufsform hätte möglicherweise durch eine intensivere Betreuung der Patientin verhindert beziehungsweise entscheidend abgemildert werden können. Grundvoraussetzung für eine ambulante Geburt, sollte die intensive postpartale Nachbetreuung durch eine Hebamme sein. Diese Leistung wird von den gesetzlichen und privaten Krankenkassen vergütet. Leider halten viele Frauen diese Dienstleistung für entbehrlich. Insbesondere bei Erstgebärenden (wie im vorliegenden Fall) kommt dieser Betreuung jedoch eine besondere Bedeutung zu. Der Hebamme wäre ein sich schlecht zurückbildender Uterus aufgefallen. Auch Veränderungen beim Wochenfluss wären für die Hebamme richtungsweisend gewesen und hätten sie eine gynäkologische Untersuchung veranlassen lassen. Damit hätte viel frühzeitiger die Verdachtsdiagnose einer unvollständigen Plazenta mit in utero verbliebenen Resten gestellt werden können. In diesem Fall hätte man der Patientin zu einer elektiven Curretage geraten. Bei dieser wäre höchstwahrscheinlich keine hämodynamisch relevante transfusionspflichtige Blutung entstanden. Da die Frau erst nach einigen Tagen und immer stärker werdender vaginaler Blutung mit Kollaps den Rettungsdienst alarmierte ging wertvolle Zeit verloren.

Das rettungsdienstliche Management entspricht weitgehend den zu fordernden Standards. Offen bleiben wenige Fragen, die im Sinne einer Qualitätsverbesserung diskutiert werden sollten: Hätte man in

Tab. 1 Laborwerte

\begin{tabular}{lccc} 
& $\begin{array}{c}\text { Abnahme gegen } \\
\text { 2:10 Uhr (NAW) }\end{array}$ & $\begin{array}{c}\text { Abnahme vor OP } \\
\text { gegen 2:50 Uhr }\end{array}$ & $\begin{array}{c}\text { Kontrolle im Aufwachraum } \\
\text { 5:30 Uhr }\end{array}$ \\
\hline Hb (Norm: 12-16 g/dl) & 9,2 & 6,2 & 5,4 \\
Hk (Norm: 37-47\%) & 27 & 19 & 16 \\
Thrombos (Norm: 150-400/nl) & 488 & 339 & 283 \\
Quick (Norm: 70-100\%) & 95 & 71 & 68 \\
PTT (Norm: 22-35 sec.) & 28 & 44 & 48
\end{tabular}


dieser Situation nicht schon präklinisch mehrere Zugänge legen sollen? Sollte der Patientin nicht auch Sauerstoff verabreicht werden? Dies ist im vorliegenden Fall nicht erfolgt beziehungsweise wurde nicht dokumentiert. Vielleicht hat das Rettungsteam aber auch die Nasensonde für den Weg vom NAW in die Aufnahme für entbehrlich gehalten. Dieses Vorgehen ist bequemer, aber medizinisch sicherlich nicht sinnvoll!

Unter der Verdachtsdiagnose einer atonen Uterusnachblutung hätte man bereits präklinisch über den Einsatz von Uterotonika nachdenken können. Dies wird auch in der gynäkologischen und notfallmedizinischen Standardliteratur empfohlen. In dieser Situation wäre die Gabe von Methergin ${ }^{\circledR}$ i.v. - Verfügbarkeit vorausgesetzt - denkbar und indiziert gewesen. In der lebensbedrohlichen Situation der (atonen) postpartalen Nachblutung rücken die Nebenwirkungen und Kontraindikationen sicher in den Hintergrund.

\section{Summary}

The present case history presents a late atonic uterine afterbleed, which progressed to haemorrhagic shock. The patient called in the medical emergency service only after the passage of several days and progressively more profuse vaginal bleeding that eventually led to collapse. Apparently, a confinement at home was not followed up by intensive postpartal care by a midwife - who would certainly have noticed that involution of the uterus was not regular. Also, changes in lochia would have provided important information and would have prompted an early gynaecological examination.

\section{Interessante Internet-Links \\ zum Thema \\ www.rote-liste.de \\ www.gyn.de \\ www.boehringer-ingelheim.de}

\section{Literatur}

1. Schmidt-Matthiesen et al. Gynäkologie und Geburtshilfe. Stuttgart: Schattauer Verlag, 1998

2. Distler et al. Notfälle in der Gynäkologie und Geburtshilfe. Heidelberg: Springer Verlag, 2001

3. Schneider $\mathrm{H}$ et al. Geburtshilfe. Heidelberg: Springer Verlag, 2000

4. Karow T. Pharmakologie und Toxikologie. Eigenverlag, 1999

5. Gorgaß B et al. Rettungsassistent und Rettungssanitäter. Heidelberg: Springer Verlag, 2001

6. Schneider T et al. Taschenatlas der Notfall- \& Rettungsmedizin. Heidelberg: Springer Verlag, 2003

7. Klinikleitfaden Gynäkologie und Geburtshilfe. München, Jena: Gustav Fischer Verlag, 1997

Weitere Literatur bei den Verfassern

\section{Anschrift für die Verfasser}

Michael Sroka

Institut für Anästhesiologie und

operative Intensivmedizin

Klinikum Stadt Hanau

Leimenstraße 20,63450 Hanau

\section{Alle Schriften auf CD-ROM Hahnemann satt - Homöopathie von Anfang an}

Edition „Samuel Hahnemann. Gesammelte Werke“, Reihe Digitale Bibliothek spezial, 149,90 Euro, ISBN 3-932544-75-7

Ob die Homöopathie nun Spinnerei ist oder nicht - Tatsache ist, dass ihr Erfinder

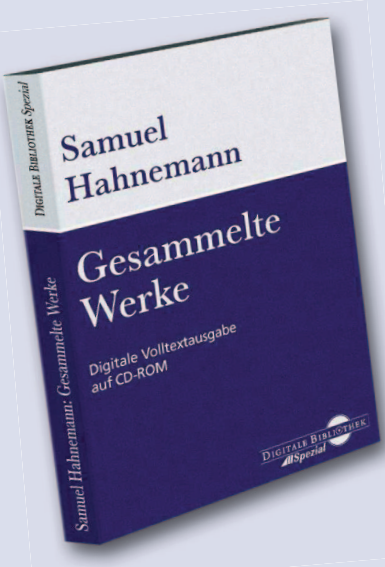
Dr. Samuel Hahnemann vor rund 200 Jahren methodische Entwicklungen in der Medizin angestoßen hat, die noch heute zentrale Errungenschaften darstellen. Beispielsweise die systematische, reproduzierbare Prüfung von Arzneimitteln an kranken und gesunden Probanden (und die standardisierte Ergebnis-Dokumentation) oder die empirische, serielle Testung völlig neuer Arzneimittel (und damit die rein experimentell begründete radikale Erweiterung unserer Pharmakopoe). Zudem hat sich Hahnemann inhaltlich-kritisch mit der Medizin seiner Zeit auseinandergesetzt - einer Medizin, deren oft anachronistisch-mittelalterliche Natur bekanntlich ebenfalls bis heute ihre Spuren hinterlässt. Wer mehr über Hahnemann erfahren will als seinen Wahlspruch „Aude sapere wage zu wissen“, kann jetzt alle seine Schriften auf einer einzigen CDROM vereint käuflich erwerben.

Die Disk enthält die vollständigen Ausgaben folgender Schriften Hahnemanns: „Apothekerlexikon“, „Versuch über ein neues Prinzip zur Auffindung der Heilkräfte der Arzneisubstanzen“, „Heilkunde der Erfahrung“, „Organon der Heilkunst“ (5. und 6. Auflage), „Reine Arzneimittellehre“ (2. und 3. Auflage) sowie „Die chronischen Krankheiten“ (2. Auflage). Insgesamt ein mehr als beeindruckender Umfang von knapp 30000 Druckseiten, dessen tatsächlichen Wert bislang wohl nur Homöopathen zu ermessen wissen. Bislang deshalb, weil der Berliner Verlag Directmedia Publishing mit der CD jetzt ein Werk vorlegt, das auch anderen Homöopathie-, Zeit- und Medizingeschichte-Interessierten genügend Studienvorlagen bietet. Neben den rein medizinischen Interessen gibt es noch einen weiteren Grund für das Studium der Hahnemann-Texte, wie es im CD-Vorwort des Berliner Essayisten Hans-Georg Soldat heißt: „Sie sind Dokumente der Menschlichkeit aus der Epoche der Aufklärung, ein Begriff, der, völlig zu Unrecht, unterdessen beinahe abschätzig gebraucht wird. Hahnemann ist ein Kind dieser Zeit, seine Geistesverwandtschaft mit den Wortführern der Aufklärung offenkundig. [...] Hahnemanns Werk ist so schließlich von erheblichem kulturhistorischen Interesse."

Die Installation des Anzeigeprogramms (lauffähig unter allen Windowsversionen ab einschließlich ' 95 , ab Herbst wird es endlich auch eine Mac-Version geben) ist schnell und problemlos möglich. Eine Kopie aller Datenverzeichnisse von CD auf Festplatte erlaubt die Nutzung des Werkes auch ohne eingelegte CD. Software-Updates und Infos stellt der Verlag unter www.digitale-bibliothek.de zur Verfügung. Die Software der Reihe Digitale Bibliothek ist anfänglich gewöhnungsbedürftig und lässt noch einige Wünsche offen. Jede Art von Basisrecherche ist jedoch rasch und problemlos möglich, dank komplexer Indexdateien stehen alle Suchergebnisse innerhalb von Sekundenbruchteilen zur Verfügung. Komplexe Suchabfragen, Wortindizes, Notizen, Übernahme von Textstellen in die Zwischenablage oder Ausdruck von bis zu 255 Seiten gleichzeitig sind ebenfalls realisierbar. Wer regelmäßig mit dem Werk arbeitet, sollte sich die vielfältigen Möglichkeiten der Software aneignen - es lohnt sich.

Rainer H. Bubenzer, Hamburg 